\section{Uso de chupeta e amamentação em crianças menores de quatro meses no estado de São Paulo}

\section{Pacifier use and breast-feeding in children under four months old in the State of São Paulo}

Lilian Cristina Cotrim 1

Sonia Isoyama Venancio 2

Maria Mercedes Loureiro Escuder 3

1-3 Instituto de Saúde. Secretaria Estadual de Saúde. Rua Santo Antonio, 590. Bela Vista. São Paulo, SP, Brasil. CEP: 01.314-000. E-mail: amamunic@isaude.sp.gov.br

\begin{abstract}
Objectives: to study pacifier use prevalence and its association to feeding practices.

Methods: data collected from the "Amamentação e Municípios" ("Breast-feeding and Municipalities") project in 1999 were analyzed. The association between pacifier use and exclusive breast-feeding interruption was verified through the $\chi^{2}$ test.

Results: prevalence of the pacifier use in 22.188 children from 0 - 4 months was high $(61,3 \%)$ and varied from $32,8 \%$ to $78,4 \%$ in 111 cities. Early pacifier introduction was noted $(53,9 \%$ in children less than one month old) as well as association between pacifier use and exclusive breast-feeding $(E B F)$ and breastfeeding $(B F)(p<0,05)$ interruption. There was a higher prevalence of bottle-feeding among pacifier users.

Conclusions: it is necessary to increase the number of protective, promotion and supportive actions to breast-feeding to reduce the high pacifier prevalence determined by this study. Child Friendly Hospital Initiatives should be included.
\end{abstract}

Key words Breast feeding, Infant care, Bottle feeding

\section{Resumo}

Objetivos: estudar a prevalência do uso da chupeta e sua associação com as práticas alimentares.

Métodos: analisado o banco de dados de 1999 do projeto "Amamentação e Municípios". A associação entre o uso de chupeta e interrupção do aleitamento materno exclusivo (AME) e aleitamento materno (AM) foi verificada através do teste de $\chi^{2}$.

Resultados: a prevalência do uso de chupeta em 22.188 menores de quatro meses foi alta (61,3\%) $e$ variou de 32,8\% a 78,4\% nos 111 municípios estudados. Observou-se a introdução precoce da chupeta (53,9\% em menores de um mês) e associação entre o uso da chupeta e interrupção do aleitamento materno exclusivo e aleitamento materno ( $p<0,05)$. Houve uma maior prevalência de uso de mamadeira entre aquelas crianças que usavam chupeta.

Conclusões: é preciso aumentar o número de ações de proteção, promoção e apoio ao aleitamento materno para que se reduza a alta prevalência de uso de chupeta evidenciada neste estudo. Uma das ações que pode contribuir para isso é a Iniciativa Hospital Amigo da Criança.

Palavras-chave Aleitamento materno, Cuidado do lactente, Alimentação artificial 


\section{Introdução}

Estudos científicos têm mostrado os benefícios da amamentação para a sobrevivência, saúde e nutrição infantis, para a saúde materna, bem como seu papel no espaçamento das gestações. 1,2

A maior parte da população civilizada perdeu o hábito de amamentar ou passou a praticá-lo por um tempo inadequado, segundo Van der Lann (1995: 5):3 "A amamentação deixou de ser vital ao ser humano no momento em que o avanço tecnológico que este adquiriu possibilitou a sobrevivência de crianças sem a obrigação de mamar no peito. Logicamente que foi o avanço industrial alcançado pela civilização nos últimos 150-300 anos que nos permitiu esta 'evolução'. Alimentos processados, mamadeiras, chupetas e mordedores são artifícios usados usualmente para substituir ou compensar as funções naturais ignoradas ou deturpadas".

As habilidades motoras orais dos recém-nascidos, as quais estão intimamente relacionadas à alimentação, e dentre estas a função de sucção, têm grande importância na alimentação dos recém-nascidos. Contribuem para o desenvolvimento global (crescimento e saúde), como também para o desenvolvimento do sistema sensório motor oral (SSMO), ou seja, dos órgãos fonoarticulatórios (OFAs): lábios, língua, mandíbula, palatos mole e duro, arcadas dentárias, dentes e musculatura oral e das funções de mastigação, deglutição e respiração, favorecendo o equilíbrio entre as estruturas.4-7 No desenvolvimento do SSMO adequado/harmonia facial do recém-nascido, é importante a normalização do "retrognatismo natural", pois a mandíbula do recémnascido possui uma diferença de cinco a oito milímetros, e até mesmo de $12 \mathrm{~mm}$ em relação à maxila. 8 É através do mecanismo da amamentação, descrito por Proença, 7 que se dá este desenvolvimento. Proença ${ }^{7}$ e Marchesan ${ }^{9}$ referem que a amamentação deve ser mantida pelo menos até o sexto mês, a fim de garantir o desenvolvimento da tonicidade orofacial.

Neste mecanismo de sucção realizado pela amamentação o desenvolvimento das funções e funcionalidade dos OFAs do recém-nascido são favorecidos: erupção dos dentes e adequada oclusão, mastigação efetiva, habilidades orais permitindo a adequada transição alimentar, assim como, a deglutição para o padrão adulto e articulação correta dos sons da fala. 6

Souza e Wertzner 10 citam alguns dos fatores que podem desencadear ou estar associados ao desenvolvimento de uma desordem do SSMO, provocando sua ocorrência e/ou permanência são: hábitos ali- mentares na primeira infância, hábitos inadequados de sucção ou qualquer outro hábito oral. O uso de mamadeira exerce influência no sistema sensório motor oral, pela produção de um trabalho muscular menor, sendo por vezes até antifisiológico.9,10 A mamadeira faz com que haja uma diminuição da ação mandibular, provocando uma sucção com movimentos de aspirar com a língua, lábios e bochechas, e isso pode levar a língua a pressionar o bico da mamadeira contra o palato, gerando conseqüentemente um palato ogival. ${ }^{9}$

Estudos relatam que a chupeta pode influenciar negativamente o aleitamento materno, ocasionando: confusão de bicos, 11,12 e diminuição da freqüência das mamadas, 13 podendo resultar o desmame precoce.13,14 Há também os aspectos negativos associados à saúde da criança, tais como: o risco de asma, vômito, febre, otalgia, 15 diarréia, 15,16 cólica, 16 enteroparasitose, ${ }^{17,18}$ ação da N-nitrosamina (substância potente em agente cancerígeno, embriopático, teratrogênico e mutagênico contida nos bicos artificiais). 19

Em 1990, representantes de diversos países produziram o documento "Amamentação nos anos 90: uma iniciativa global", que tratava de um conjunto de metas chamado "Declaração de Innocenti" (Sokol; 1999: 7-11). ${ }^{20}$ Essa Declaração, que tem sido a base para as recomendações internacionais sobre práticas alimentares no primeiro ano de vida, enfatiza a recomendação do aleitamento materno exclusivo até quatro a seis meses de idade, associado a outros alimentos até os dois anos. Mais recentemente o aleitamento materno exclusivo passou a ser recomendado até sexto mês. ${ }^{21}$

Venancio e Monteiro22 mostraram que a amamentação no Brasil tem aumentado (sua duração mediana passou de 2,5 meses para 5,5 meses durante o período de 1975 a 1989), porém a prática da amamentação exclusiva era pouco freqüente na década de $80,(3,6 \%$ em crianças de zero a quatro meses). Um estudo recente 23 realizado em capitais brasileiras mostrou prevalência em torno de $20 \%$ de amamentação exclusiva em menores de quatro meses, o que mostra que esta prática está longe de ser universal em nosso país. Em se tratando do Estado de São Paulo, o Aleitamento Materno exclusivo (AME) raramente alcançou índices superiores à $30 \%$, nesta faixa etária. 24

Algumas ações foram criadas para promover o aleitamento materno, dentre as quais se destacam: Iniciativa Hospital Amigo da Criança (IHAC) e Norma Brasileira de Comercialização de Alimentos ao Lactentes (NBCAL).

Em 1991, a Organização Mundial da Saúde 
(OMS) e o Fundo das Nações Unidas para a Infância (UNICEF) lançam a IHAC, idealizada para apoiar, proteger e promover o aleitamento materno. Propõem um conjunto de medidas para atingir as metas contidas na "Declaração de Innocenti", denominadas "Dez passos para o sucesso do aleitamento materno".25 Dentre os dez passos, destaca-se a orientação do não uso de bicos artificiais ou chupetas por crianças amamentadas.

A NBCAL foi lançada em 1988, com o "...objetivo de contribuir para a adequada nutrição dos lactentes e defendê-los dos riscos associados à não amamentação ou desmame precoce, proteger e incentivar a amamentação" (Sokol; 1999: 194).20

Tendo em vista o impacto negativo do uso da chupeta e mamadeira sobre amamentação, este estudo tem por objetivos: descrever a prevalência do uso de chupeta em crianças menores de quatro meses em municípios do Estado de São Paulo, descrever o uso de chupeta segundo os diferentes padrões de aleitamento infantil e, verificar a associação entre os usos de chupeta e mamadeira na alimentação infantil.

\section{Métodos}

Foram utilizados dados de 1999 do Projeto "Amamentação e Municípios: avaliação de práticas alimentares no primeiro ano de vida em dias nacionais de vacinação", coordenado pelo Instituto de Saúde da Secretaria de Saúde do Estado de São Paulo (SES, SP). O projeto capacita municípios para a realização de inquéritos epidemiológicos nos municípios do Estado, de forma rápida e pouco dispendiosa. A participação dos municípios é espontânea, evidenciando disponibilidade para a realização da pesquisa, participação em um treinamento (duração de oito horas) e interesse em implementar ações de promoção da amamentação. Os municípios de pequeno porte realizam a pesquisa em caráter universal (em 100\% dos menores de um ano) e os municípios grandes utilizam amostragem por conglomerados. 26

O instrumento para coleta de dados proposto utiliza perguntas fechadas e inclui questões sobre a alimentação da criança nas últimas 24 horas, uso de chupeta e mamadeira, seguindo as recomendações da World Health Organization (WHO) para inquéritos sobre amamentação.

Os municípios participantes recebem um programa, "Amamentação e município" (AMAMUNIC), 27 desenvolvido para o armazenamento e análise dos dados o que facilita o gerenciamento dos dados por parte dos municípios.

A distribuição geográfica da prevalência do uso de chupeta dos municípios participantes é apresentada em um mapa elaborado através do programa Tabwin28 (Figura 1). 
Mapa com as proporções do uso de chupeta distribuídas pelo municípios do estado de São Paulo, Brasil. (Projeto "Amamentação e Municípios", 1999).

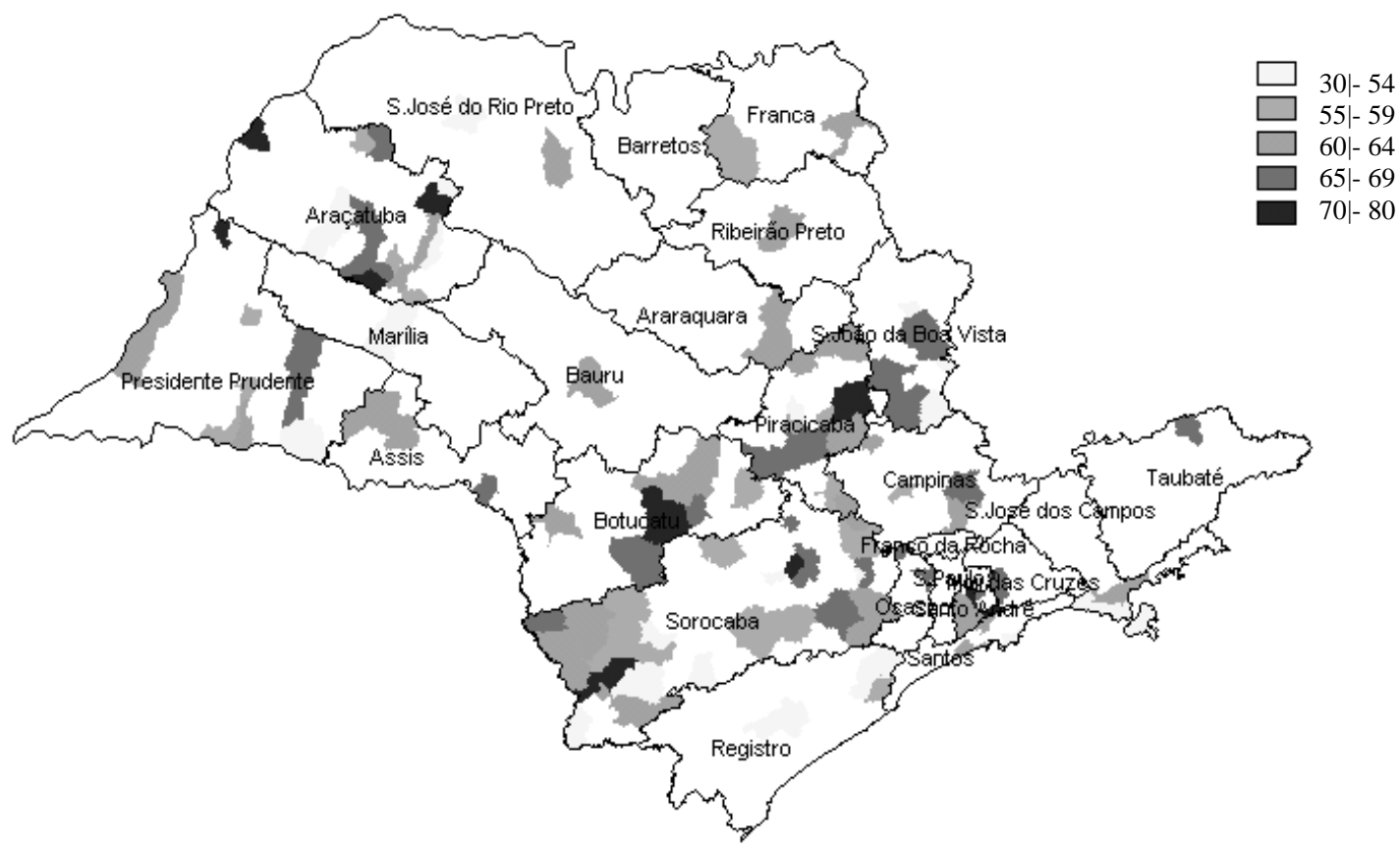

As associações entre o uso de chupeta e os diferentes padrões de amamentação e do uso de chupeta e mamadeira foram analisadas através do teste de qui-quadrado com nível de significância de 0,05 , utilizando-se o programa SPSS. 29 São também apresentados os valores de odds ratio e os respectivos intervalos de confiança para analisar as chances de interrupção do aleitamento materno (AM), aleitamento materno exclusivo, e amamentação entre crianças que usam e não usam chupeta.

A comparação entre a proporção de crianças em AME em diferentes idades nos grupos de crianças que usam e não usam chupeta é apresentada por meio da média móvel, obtendo-se a média entre as porcentagem de três pontos 30 (idade em quinzenas).

\section{Resultados}

O banco de dados soma 22.188 registros de crianças menores de quatro meses, distribuídos entre $111 \mathrm{mu}-$ nicípios do Estado de São Paulo. Algumas questões sem informação ou com dados inconsistentes foram excluídas das tabelas.

Na Figura 1 apresenta-se a distribuição da prevalência do uso de chupeta nos municípios participantes. Esta prevalência variou entre $32,8 \%$ e $78,4 \%$; entre as 22.188 crianças, $61,3 \%$ fizeram o uso de chupeta nas últimas $24 \mathrm{hs}$. Das 4.549 crianças menores de um mês, 53,9\% usavam chupeta.

Embora exista uma distribuição heterogênea do uso de chupeta nos municípios estudados, cerca de 69 deles apresentaram porcentagens elevadas (60 a $80 \%$ ), e no município de menor prevalência mais de $1 / 3$ das crianças utilizavam chupeta. A heterogeneidade aparece até mesmo entre municípios de mesma região administrativa.

Na Tabela 1 verifica-se associação significativa entre uso de chupeta e interrupção do AME ( $\mathrm{p}<$ $0,05)$. Dentre as crianças que usam chupeta, há mais chance de interrupção do AME quando comparadas àquelas que não usam chupeta $(\mathrm{OR}=3,26)$. Da mes- 
ma forma existe associação significativa do uso de chupeta e desmame completo (Tabela 2), sendo que as crianças que usam chupeta também apresentam mais chances de interrupção do AM quando comparadas com as que não usam chupeta $(\mathrm{OR}=5,99)$.

\section{Tabela 1}

Associação uso de chupeta e interrupção do aleitamento materno exclusivo. São Paulo, SP, Brasil, 1999.

\begin{tabular}{|c|c|c|c|c|c|}
\hline \multirow[b]{2}{*}{ Uso de chupeta } & \multicolumn{2}{|c|}{$\begin{array}{l}\text { Interrupção do aleitamento } \\
\text { materno exclusivo (AME) }\end{array}$} & \multirow[b]{2}{*}{ Total } & \multirow[b]{2}{*}{ OR } & \multirow[b]{2}{*}{ IC95\% } \\
\hline & Sim & Não & & & \\
\hline \multicolumn{6}{|l|}{ Sim } \\
\hline \multirow[t]{2}{*}{$\mathrm{n}$} & 11.904 & 1.616 & 13.520 & & \\
\hline & & & & 3,26 & $3,00-3,50$ \\
\hline$\%$ & 88,0 & 12,0 & 100,0 & & \\
\hline Não & & & & & $(p<0,05)$ \\
\hline $\mathrm{n}$ & 5.912 & 2.613 & 8.525 & & \\
\hline$\%$ & 69,3 & 30,7 & 100,0 & & \\
\hline \multicolumn{6}{|l|}{ Total } \\
\hline $\mathrm{N}$ & 17.816 & 4.229 & 22.045 & & \\
\hline$\%$ & 80,8 & 19,2 & 100,0 & & \\
\hline
\end{tabular}

Tabela 2

Associação uso de chupeta e interrupção do aleitamento materno. São Paulo, SP, Brasil, 1999.

\begin{tabular}{|c|c|c|c|c|c|}
\hline \multirow[b]{2}{*}{ Uso de chupeta } & \multicolumn{2}{|c|}{$\begin{array}{c}\text { Interrupção do aleitamento } \\
\text { materno (AM) }\end{array}$} & \multirow[b]{2}{*}{ Total } & \multirow[b]{2}{*}{ OR } & \multirow[b]{2}{*}{ IC95\% } \\
\hline & Sim & Não & & & \\
\hline \multicolumn{6}{|l|}{ Sim } \\
\hline \multirow[t]{2}{*}{$\mathrm{n}$} & 4.138 & 9.300 & 13.438 & & \\
\hline & & & & 5,99 & $5,50-6,60$ \\
\hline$\%$ & 30,8 & 69,2 & 100,0 & & \\
\hline Não & & & & & $(p<0,05)$ \\
\hline $\mathrm{n}$ & 589 & 7.921 & 8.510 & & \\
\hline$\%$ & 6,9 & 93,1 & 100,0 & & \\
\hline \multicolumn{6}{|l|}{ Total } \\
\hline $\mathrm{N}$ & 4.727 & 17.221 & 21.948 & & \\
\hline$\%$ & 21,5 & 78,5 & 100,0 & & \\
\hline
\end{tabular}


Verifica-se também maior prevalência do uso de mamadeira entre as crianças que usam chupeta $(64,2 \%)$ quando comparadas com as que não usam
$(39,8 \%)$, sendo constatada a significância estatística desta associação no teste do $\chi^{2}(\mathrm{p}<0,05)$ e no $o d d s$ ratio de 2,71(Tabela 3 ).

\section{Tabela 3}

Associação entre uso de chupeta e uso de mamadeira. São Paulo, SP, Brasil, 1999.

\begin{tabular}{|c|c|c|c|c|c|}
\hline \multirow[b]{2}{*}{ Uso de chupeta } & \multicolumn{2}{|c|}{ Uso de mamadeira } & \multirow[b]{2}{*}{ Total } & \multirow[b]{2}{*}{ OR } & \multirow[b]{2}{*}{ IC95\% } \\
\hline & Sim & Não & & & \\
\hline \multicolumn{6}{|l|}{ Sim } \\
\hline \multirow[t]{2}{*}{$\mathrm{n}$} & 8.544 & 4.772 & 13.316 & & \\
\hline & & & & 2,71 & $2,60-2,90$ \\
\hline$\%$ & 64,2 & 35,8 & 100,0 & & \\
\hline \multicolumn{6}{|l|}{ Não } \\
\hline $\mathrm{n}$ & 3.335 & 5.046 & 8.381 & \multicolumn{2}{|r|}{$(p<0,05)$} \\
\hline$\%$ & 39,8 & 60,2 & 100,0 & & \\
\hline \multicolumn{6}{|l|}{ Total } \\
\hline $\mathrm{N}$ & 11.879 & 9.818 & 21.697 & & \\
\hline$\%$ & 54,7 & 45,3 & 100,0 & & \\
\hline
\end{tabular}

A partir do gráfico de média móvel observa-se em todas as idades maior porcentagem de crianças em AME dentre as que não fazem uso de chupeta
(Figura 2), sendo que no primeiro mês verificou-se uma diferença mais acentuada, de 20 pontos percentuais.

\section{Figura 2}

Média móvel segundo a proporção de crianças que estão em aleitamento materno exclusivo em relação ao uso da chupeta. São Paulo, SP, Brasil, 1999.

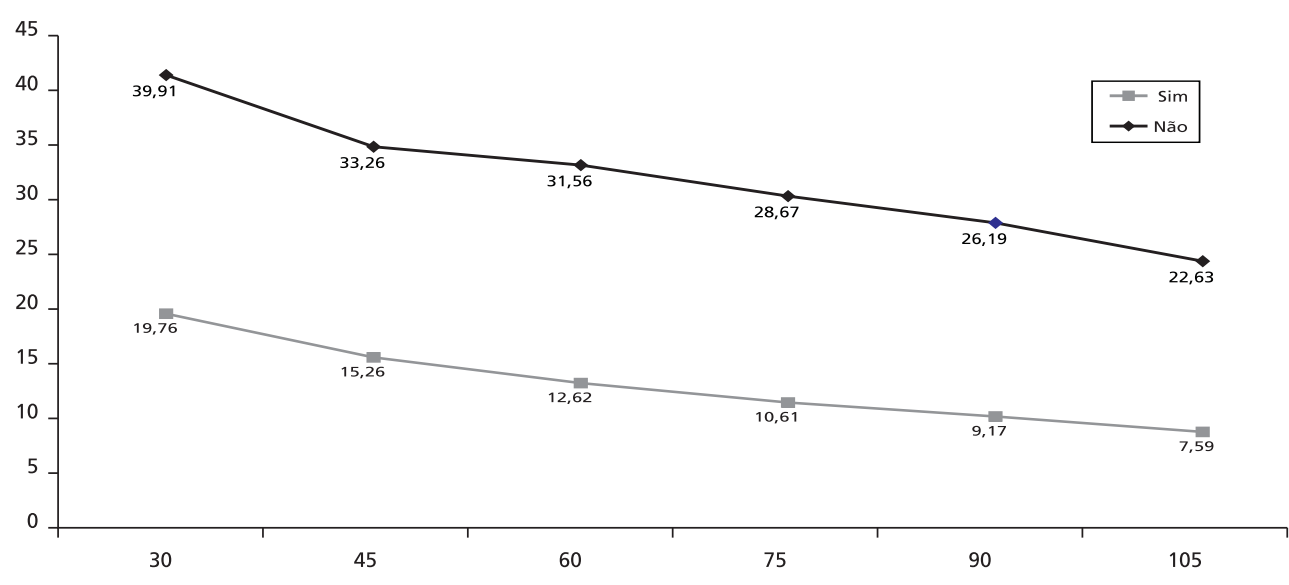




\section{Discussão}

Neste estudo pôde ser visualizada a heterogeneidade da prevalência do uso da chupeta em 111 municípios do Estado de São Paulo.

Verificou-se que a prevalência do uso de chupeta em crianças menores de quatro meses é elevada, assim como a introdução "precoce" de chupeta em nosso meio, corroborando estudos nacionais e internacionais. $15,31,32$

Embora o desenho (transversal) deste estudo não seja o mais adequado para estabelecer relações causais, a forte associação entre o uso de chupeta e interrupção do AME indica a necessidade de realização de estudos de seguimento que comprovem a hipótese da chupeta interferir positivamente para o desmame. A possível interferência do uso de chupeta sobre o aleitamento materno identificado neste estudo foi também descrita por Victora et al., ${ }^{31}$ os quais relataram $65 \%$ de interrupção do AM ao seis meses em um grupo de crianças que usaram chupeta com um mês de idade, contra $24 \%$ no grupo que não usava. Os mesmos autores, estudando mães de baixa renda de uma cidade de médio porte no sul do Brasil, verificaram que o uso da chupeta estava associado a um maior risco de parar de amamentar aos três e seis meses de idade. ${ }^{31}$ Richard e Alade 12 relatam que a interferência do uso da chupeta quando a amamentação não está estabelecida resulta no desmame precoce na maioria dos casos.

Apesar das evidências dos efeitos nocivos do uso da chupeta existem alguns estudos na literatura que apontam benefícios, como: a prevenção da morte súbita, (ainda em discussão e bastante questionada), 32 inibição da hiperatividade e modulação do desconforto do recém-nascido. 33 Sendo aplicado de maneira seletiva, em populações neonatais específicas e apenas como medida coadjuvante para o tratamento da dor do recém-nascido, não tendo propriedade analgésica intrínseca, evita que o bebê torne-se chupador de dedo33 e acalma o bebê.33,34 Isto pode levar a diferentes condutas por parte dos profissionais de saúde.

A prevalência maior do uso de mamadeira entre as crianças que usam chupeta sugere o desmame dessas mas o estudo de Howard et al. 13 refere que o uso freqüente da chupeta interfere significativamente na amamentação acarretando o desmame precoce, estando ou não associado com a introdução da mamadeira.

A literatura traz os fatores que influenciam o uso de chupeta, tais como: as questões culturais, a insegurança da mãe em amamentar, bem como as dificuldades e problemas na amamentação, 31 a interferência da mídia 34 e conduta de alguns profissionais quanto as orientações sobre o uso da chupeta. ${ }^{5}$ Este hábito poderia ser evitado com a capacitação dos profissionais da saúde, do meio hospitalar e de unidades básicas de saúde, para o manejo da amamentação; o método mãe-canguru; o monitoramento da Norma Brasileira de Comercialização de Alimento ao Lactente e a Iniciativa Hospital Amigo da Criança.

A Iniciativa Hospital Amigo da Criança abarca várias das questões citadas acima, com o estudo de Aarts et al. ${ }^{32}$ verificou-se a sua contribuição para a temática, ao ser constatada uma súbita queda do uso da chupeta em menores de uma semana em crianças que nasceram em Hospitais Amigo da Criança, ao desaconselharem o uso de bicos artificiais ou chupetas no período pós-parto.

Há indícios portanto, de que a ampliação dos Hospitais Amigos da Criança no Estado (atualmente em número de 14) poderia reduzir a freqüência do uso de chupeta e aumentaria o aleitamento materno exclusivo no Estado de São Paulo.

\section{Referências}

1. WHO (World Health Organization). Breast-feeding: the technical basis and recommendations for action. Geneva: WHO; 1993.

2. Akré J. Alimentação infantil: bases fisiológicas. São Paulo: IBFAN (Internacional Baby Food Network); 1994.

3. Van der Laan CDT. A importância da amamentação natural no desenvolvimento facial. Pró-fono 1995; 7: 3-5.
4. Jacintho I. Estimulação de sucção para RN de alto risco. In: Marchesan IQ, organizador. Fundamentos em fonoaudiologia: aspectos clínicos da motricidade oral. Rio de Janeiro: Guanabara-Koogan; 1998. p. 7-11.

5. Mascarenhas CF. Sucção de chupeta: qual a razão da utilização da chupeta no recém-nascido. Fono Atual 1999; 3: $25-8$.

6. Xavier C. Avaliação da alimentação de RN em fase de hos- 
pitalização. Pró-fono 1995; 7: 69-74

7. Proença MG. Sistema sensório motor oral. In: Kudo AM, Marcondes E, Lins L, Moriayama LT, Guimarães MLLG, Juliani RCTP, Pierri SA. Fisioterapia, fonoaudiologia e terapia ocupacional em pediatria. Rio de Janeiro: Sarvier; 1994. p. 116-24

8. Tanigute CC. Desenvolvimento das funções estomatognáticas. In: Marchesan IQ. Fundamentos em fonoaudiologia: aspectos clínicos da motricidade oral. Rio de Janeiro: Guanabara-Koogan; 1998. p. 13-41.

9. Marchesan IQ. Uma visão compreensiva das práticas alimentares: a influência da alimentação no crescimento e desenvolvimento craniofacial e nas alterações miofuncionais São Paulo: Pancast; 1998.

10. Souza MA, Wertzner HF. Uma análise de crianças normais de 3 a 8 anos In: Limongi SCO, organizador. Fonoaudiologia e pesquisa. São Paulo: Lovise; 1998. (Atualidades em Fonoaudiologia, v. 4)

11. Neifert M, Lawrence R, Seacat JRN. Nipple confusion: toward a formal definition. Pediatrics 1997; 130: 125-9.

12. Richard L, Alade MO. Breastfeeding and the pacifiers use. Birth 1997; 24: 116-20.

13. Howard CR, Howard FM, Lanphear EB, Eberlu S, Lawerence RA. The effects of early pacifier use on breastfeeding duration. Pediatrics 1999; 103 [serial online] Available from: <http:llwww.pediatrics.org/cgi/content/full/ 103/3/e33> [2000 Dez 11]

14. Victora CG, Tomasi E, Olinto MTA, Barros FC. Use of pacifiers and breastfeeding duration. Lancet 1993; 341: 404-6.

15. North K, Fleming P, Golding J. Pacifier use and morbidity in the first six month of life. Pediatrics 1999; 103. [serial on line] Available from: <http: Ilwww.pediatrics.org/cgi/ content/full/103/3/e34> [2000 Dez 11]

16. Tomasi E. Uso de chupeta: padrões, contaminações e associações com diarréia [dissertação mestrado]. Pelotas: Departamento de Medicina Social, Faculdade de Medicina da Universidade Federal de Pelotas; 1993.

17. Silva GAP.O uso de chupetas contribui para uma maior ocorrência de enteroparasitose? J Pediatr [Rio de Janeiro] 1997; 73: 2-3.

18. Pedroso RS, Siqueira RV. Pesquisa de cistos de protozoários, larvas e ovos de helmintos em chupetas. J Pediatr [Rio de Janeiro] 1997; 73: 21-5.

19. Glória MBAN. Nitrosaminas em bicos de mamadeiras e chupetas: documentação do mês sobre amamentação. São Paulo: Instituto de Saúde da Secretaria Estadual de Saúde; 1999.

20. Sokol EJ. Em defesa da amamentação: manual para implementar o código internacional de mercadização de sub- stitutos do leite materno São Paulo: IBFAN (Internacional Baby Food Network); 1999.

21. WHO (World Health Organization). Infant and young child nutrition. In: LIV World Health Assembly. Geneva; 2001. (WHA 54.2). Available from: URL: $\langle\mathrm{http}: \backslash \backslash$ www.who.int/gb/EB_WHA/PDF/WHA54/ ea54r2.pdf $>$ [2001 May 28]

22. Venancio SI, Monteiro CA. A tendência da prática da amamentação no Brasil nas décadas de 70 e 80 . Rev Bras Epidemiol 1998; 1: 40-9

23. Ministério da Saúde. Secretaria de Políticas Públicas. Área de Saúde da Criança. Prevalência de aleitamento materno nas capitais brasileiras e no Distrito Federal. Brasília, DF: Ministério da Saúde; 2001.

24. Venancio SI, Escuder, MML, Kitoko P, Rea MF, Monteiro CA. Freqüência e determinantes do aleitamento materno em municípios do Estado de São Paulo. Rev Saúde Pública 2002; 36: 313-8.

25. OMS (Organización Mundial de la Salud). Proteccion, promocion y apoyo de la lactancia natural: la función especial de los serviços de maternidad. Ginebra: OMS 1989.

26. Silva NN. Amostragem probabilística: um curso introdutório. São Paulo: Universidade de São Paulo; 1998.

27. Instituto de Saúde. Intellisoft. Amamunic: amamentação e municípios [programa para windows]. Versão 1.0. São Paulo: Instituto de Saúde; 1999.

28. Ministério da Saúde. DATASUS. Tab Win - Tab para windows [programa de computador]. Versão 2.0. Brasília, DF: Ministério da Saúde; 2001. Disponível em <http: Ilwww.datasus.gov.br> [2001 mar 1].

29. SPSS (Statistical Package for the Social Sciences) [program for windows]. Versão 8.0.0. Chicago: SPSS; 1997.

30. OPS (Organización Panamericana de la Salud), OMS (Organización Mundial de la Salud). Indicadores para evaluar las praticas de lactancia materna. Ginebra: OPS; 1991

31. Victora CG, Behague DP, Barros FC, Olinto MTA, Weiderpass E. Pacifier use and short breastfeeding duration: cause, consequence, or coincidence? Pediatrics 1997; 99 : 445-53.

32. Aarts C, Hornell A, Kylberg E, Hofvander Y, GebreMedlin M. Breastfeeding patterns in relation to thumb sucking and pacifier use. Pediatrics [on line] 1999; 104 Available from: URL: <http:Ilwww.pediatrics.org/cgi/ content/full/104/4/e50> [2000 Dez 11]

33. Guinsburg R. Avaliação e tratamento da dor no recémnascido. J Pedriatr [Rio de Janeiro] 1999; 75 Supl 3 149-60.

34. Panhoca I, Paffaro CA, Mello JS. Chupeta e mamadeira, um tema de fonoaudiologia. Rev Fono Atual 1999; 3: 11-7.

Recebido em 25 de junho de 2002

Versão final reapresentada em 3 de agosto de 2002

Aprovado em 9 de setembro de 2002 